\title{
On 10-Centralizer Groups of Odd Order
}

\author{
Z. Foruzanfar and Z. Mostaghim \\ School of Mathematics, Iran University of Science and Technology, Narmak, Tehran 1684613114, Iran
}

Correspondence should be addressed to Z. Mostaghim; mostaghim@iust.ac.ir

Received 17 February 2014; Accepted 12 March 2014; Published 1 April 2014

Academic Editors: J. Rada and S. Yang

Copyright (C) 2014 Z. Foruzanfar and Z. Mostaghim. This is an open access article distributed under the Creative Commons Attribution License, which permits unrestricted use, distribution, and reproduction in any medium, provided the original work is properly cited.

Let $G$ be a group, and let $|\operatorname{Cent}(G)|$ denote the number of distinct centralizers of its elements. A group $G$ is called $n$-centralizer if $|\operatorname{Cent}(G)|=n$. In this paper, we investigate the structure of finite groups of odd order with $|\operatorname{Cent}(G)|=10$ and prove that there is no finite nonabelian group of odd order with $|\operatorname{Cent}(G)|=10$.

\section{Introduction}

Throughout this paper all groups mentioned are assumed to be finite, and we will use usual notation; for example, $C_{n}$ denotes the cyclic group of order $n$, and $C_{n} \rtimes C_{p}$ denotes the semidirect product of $C_{n}$ and $C_{p}$ with normal subgroup $C_{n}$, where $n$ is a positive integer and $p$ is a prime. For a group $G$, $Z(G)$ denotes the center of $G$, and $\operatorname{Cent}(G)=\left\{C_{G}(x) \mid x \in\right.$ $G\}$, where $C_{G}(x)$ is the centralizer of the element $x$ in $G$; that is, $C_{G}(x)=\{y \in G \mid x y=y x\}$. A group $G$ is a $C A$-group if $C_{G}(x)$ is abelian for every $x \in G \backslash Z(G)$. Many authors have studied the influence of $|\operatorname{Cent}(G)|$ on finite group $G$ (see [19]). It is clear that a group $G$ is 1-centralizer if and only if it is abelian. In [6] Belcastro and Sherman proved that there is no $n$-centralizer group for $n=2,3$. They also proved that $G$ is 4-centralizer if and only if $G / Z(G) \cong C_{2} \times C_{2}$, and $G$ is 5-centralizer if and only if $G / Z(G) \cong C_{3} \times C_{3}$ or $S_{3}$. In [2] Ashrafi proved that if $G$ is 6-centralizer, then $G / Z(G) \cong D_{8}$, $A_{4}, C_{2} \times C_{2} \times C_{2}$, or $C_{2} \times C_{2} \times C_{2} \times C_{2}$. In [1] Abdollahi et al. proved that $G$ is 7-centralizer if and only if $G / Z(G) \cong C_{5} \times C_{5}$, $D_{10}$ or $\left\langle x, y \mid x^{5}=y^{4}=1, y^{-1} x y=x^{3}\right\rangle$. They also proved that if $G$ is 8-centralizer, then $G / Z(G) \cong C_{2} \times C_{2} \times C_{2}, A_{4}$ or $D_{12}$.

Our main result is as follows.

Theorem 1. There is no finite nonabelian group G of odd order with $|\operatorname{Cent}(G)|=10$.

\section{Preliminary Results}

By [1], a cover $\Gamma$ for a group $G$ is a collection of proper subgroups whose union is the whole $G$. We use the term $n$-cover for a cover with $n$ members. A cover is called irredundant if no proper subcollection is also a cover. A cover is called a partition with kernel $k$ if the intersection of pairwise members of the cover is $k$. Neumann in [10] obtained a uniform bound for the index of the intersection of an irredundant $n$-cover in terms of $n$, and Tomkinson [11] improved this bound. For a natural number $n$, let $f(n)$ denote the largest index $|G: D|$, where $G$ is a group with an irredundant $n$-cover whose intersection of all of them is $D$. We know that $f(3)=4, f(4)=9, f(5)=16$, and $f(6)=36$ (see [12-15], resp.). Now we present some lemmas and propositions that will be used in the proof of Theorem 1 .

Lemma 2 (Lemma 3.3 of [11]). Let $M$ be a proper subgroup of the finite group $G$, and let $H_{1}, H_{2}, \ldots, H_{k}$ be subgroups of $G$ with $\left|G: H_{i}\right|=\beta_{i}$ and $\beta_{1} \leq \cdots \leq \beta_{k}$. If $G=M \cup H_{1} \cup \cdots \cup H_{k}$, then $\beta_{1} \leq k$. Furthermore, if $\beta_{1}=k$, then $\beta_{1}=\beta_{2}=\cdots=$ $\beta_{k}=k$ and $H_{i} \cap H_{j} \leq M$ for any two distinct $i$ and $j$.

Definition 3 (Definition 2.1 of [1]). A nonempty subset $X=$ $\left\{x_{1}, \ldots, x_{r}\right\}$ of a finite group $G$ is called a set of pairwise noncommuting elements if $x_{i} x_{j} \neq x_{j} x_{i}$ for all distinct $i, j \in$ $\{1, \ldots, r\}$. A set of pairwise noncommuting elements of $G$ is 
said to have maximal size if its cardinality is the largest one among all such sets.

Remark 4. Let $G$ be a finite group, and let $\left\{x_{1}, \ldots, x_{r}\right\}$ be a set of pairwise noncommuting elements of $G$ having maximal size. Then

(1) $\left\{C_{G}\left(x_{i}\right) \mid i=1, \ldots, r\right\}$ is an irredundant $r$-cover with the intersection $Z(G)=\cap_{i=1}^{r} C_{G}\left(x_{i}\right)$ (see Theorem 5.1 of [11]);

(2) $|G / Z(G)| \leq f(r)$ (see Corollary 5.2 of [11]);

(3) $f(3)=4, f(4)=9, f(5)=16, f(6)=36$, and $f(7)=$ 81 (see [12-16], resp.);

(4) let $G$ be a group such that every proper centralizer in $G$ is abelian. Then for all $a, b \in G \backslash Z(G)$ either $C_{G}(a)=$ $C_{G}(b)$ or $C_{G}(a) \cap C_{G}(b)=Z(G)$.

If $z \in\left(C_{G}(a) \cap C_{G}(b)\right) \backslash Z(G)$, then $C_{G}(z)$ contains both $C_{G}(a)$ and $C_{G}(b)$, since $C_{G}(a)$ and $C_{G}(b)$ are abelian. Since $z$ is not in $Z(G), C_{G}(z) \leq C_{G}(a)$ and $C_{G}(z) \leq C_{G}(b)$. Thus, $C_{G}(z)=C_{G}(a)=C_{G}(b)$. Hence, in such a group $G,\left\{C_{G}(x) \mid\right.$ $x \in G \backslash Z(G)\}$ forms a partition with kernel $Z(G)$. It follows that $\left\{C_{G}(x) / Z(G) \mid x \in G \backslash Z(G)\right\}$ forms a partition whose kernel is the trivial subgroup (see also Proposition 1.2 of [17]).

Lemma 5 (Lemma 2.4 of [1]). Let $G$ be a finite nonabelian group, and let $\left\{x_{1}, \ldots, x_{r}\right\}$ be a set of pairwise noncommuting elements of $G$ with maximal size. Then
(1) $r \geq 3$
(2) $r+1 \leq|\operatorname{Cent}(G)|$;
(3) $r=3$ if and only if $|\operatorname{Cent}(G)|=4$;
(4) $r=4$ if and only if $|\operatorname{Cent}(G)|=5$.

Proposition 6 (Proposition 2.5 of [1]). Let $G$ be a finite group, and let $X=\left\{x_{1}, \ldots, x_{r}\right\}$ be a set of pairwise noncommuting elements of $G$ having maximal size.

(a) If $|\operatorname{Cent}(G)|<r+4$, then

(1) for each element $x \in G, C_{G}(x)$ is abelian if and only if $C_{G}(x)=C_{G}\left(x_{i}\right)$ for some $i \in\{1, \ldots, r\}$;

(2) if $C_{G}\left(x_{i}\right)$ is a maximal subgroup of $G$ for some $i \in\{1, \ldots, r\}$, then $Z(G)=C_{G}\left(x_{i}\right) \cap C_{G}\left(x_{j}\right)$ for all $j \in\{1, \ldots, r\} \backslash\{i\}$. In particular, if $\left|G: C_{G}\left(x_{1}\right)\right| \leq$ $\left|G: C_{G}\left(x_{2}\right)\right| \leq 2$, then $|\operatorname{Cent}(G)|=4$, and if $\mid G$ : $C_{G}\left(x_{1}\right)|\leq| G: C_{G}\left(x_{2}\right) \mid=3$, then $|\operatorname{Cent}(G)|=5$.

(b) If $|\operatorname{Cent}(G)|=r+2$, then there exists a proper nonabelian centralizer $C_{G}(x)$ which contains $C_{G}\left(x_{i_{1}}\right)$, $C_{G}\left(x_{i_{2}}\right)$, and $C_{G}\left(x_{i_{3}}\right)$ for three distinct $i_{1}, i_{2}, i_{3} \in$ $\{1, \ldots, r\}$.

(c) If $|\operatorname{Cent}(G)|=r+3$, then there exists a proper nonabelian centralizer $C_{G}(x)$ which contains $C_{G}\left(x_{i_{1}}\right)$ and $C_{G}\left(x_{i_{2}}\right)$ for two distinct $i_{1}, i_{2} \in\{1, \ldots, r\}$.

Lemma 7 (Lemma 2.6 of [1]). Let $G$ be a finite nonabelian group. Then every proper centralizer of $G$ is abelian if and only if $|\operatorname{Cent}(G)|=r+1$, where $r$ is the maximal size of a set of pairwise noncommuting elements of $G$.
Theorem 8 (Theorem 4.2 of [11]). Suppose that $G$ is covered by $n$ abelian subgroups $A_{1}, A_{2}, \ldots, A_{n}$; then

(i) if $G=\left\langle A_{1}, A_{2}\right\rangle$, then $|G / Z(G)| \leq(n-1)^{2}$;

(ii) if $\left\langle A_{1}, A_{2}\right\rangle<G$, then $|G / Z(G)| \leq 2(n-2)^{\log _{2}(n-2)}$.

Definition 9 (see [18]). A group $G$ is said to be an $n$-sum group if it can be written as the sum of $n$ of its proper subgroups and of no smaller number. One then writes $G=\sum_{r=1}^{n} H_{r}$, where, for each $r, H_{r}$ is a proper subgroup, which can be assumed to be maximal where convenient.

Theorem 10 (Theorem 1 of [18]). If $G=\sum_{r=1}^{n} H_{r}$, then $|G| \leq$ $\sum_{r=2}^{n}\left|H_{r}\right|$, with equality if and only if (a) $H_{1} H_{r}=G ; r \neq 1$ and (b) $H_{r} \cap H_{s} \subset H_{1} ; r \neq s$.

Remark 11. If $A$ and $B$ are subgroups of finite index in a group $G$, and $|G: A|$ and $|G: B|$ are relatively prime, then $G=A B$.

Proposition 12 (Proposition 2.2 of [19]). Let $p$ be the smallest prime that is dividing $|G|$. If $|G: Z(G)|=p^{3}$, then $|\operatorname{Cent}(G)|=$ $p^{2}+p+2$ or $p^{2}+2$.

Proposition 13 (Proposition 2.9 of [19]). Let $G / Z(G)$ be nonabelian, $n$ an integer, and $p$ a prime. If $G / Z(G) \cong C_{n} \rtimes C_{p}$, then $|\operatorname{Cent}(G)|=n+2$.

Now we are ready to state the main result of this paper.

\section{The Proof of Theorem 1}

In this section, we give the proof of the main theorem.

Suppose, on the contrary, that $G$ is a finite nonabelian group of odd order with $|\operatorname{Cent}(G)|=10$. Let $\left\{x_{1}, \ldots, x_{r}\right\}$ be a set of pairwise noncommuting elements of finite nonabelian group $G$ having maximal size. Then $X_{i}=C_{G}\left(x_{i}\right), 1 \leq i \leq r$, is an irredundant $r$-cover with intersection $Z(G)$. Assume that $\left|G: X_{i}\right|=\alpha_{i}$, where $\alpha_{1} \leq \cdots \leq \alpha_{r}$. Since $G$ is a 10 -centralizer group, therefore, by Lemma 5 , we have $5 \leq r \leq 9$.

Suppose $r=5$. By Remark $4,|G / Z(G)| \leq 16$ and $G=$ $X_{1} \cup X_{2} \cup \cdots \cup X_{5}$; therefore by Lemma 2, $\left|G: X_{2}\right| \leq 4$. Since $|G|$ is odd, then $\left|G: X_{2}\right|=3$. On the other hand, 3 is a divisor of $|G / Z(G)|$; therefore, we have $|G / Z(G)| \in\{9,15\}$. If $|G / Z(G)|=9$, then $|\operatorname{Cent}(G)|=5$ by $[6$, Theorem 6], which is a contradiction. Now if $|G / Z(G)|=15$, then $G$ is abelian which is not possible. It implies that $r \neq 5$.

Suppose $r=6$. By Remark $4,|G / Z(G)| \leq 36$ and $G=$ $X_{1} \cup X_{2} \cup \cdots \cup X_{6}$; therefore by Lemma 2, $\left|G: X_{2}\right| \leq 5$. If $\left|G: X_{2}\right|=5$, since 5 is a divisor of $|G / Z(G)|$, then $|G / Z(G)| \in\{15,25,35\}$. If $|G / Z(G)| \in\{15,35\}$, then $G$ is abelian, which is not possible. If $|G / Z(G)|=25$, then by $[6$, Theorem 6], $|\operatorname{Cent}(G)|=7$, a contradiction. Now suppose $\left|G: X_{2}\right|=3$; since 3 is a divisor of $|G / Z(G)|$, therefore $|G / Z(G)| \in\{9,15,21,27,33\}$. If $|G / Z(G)| \in\{15,33\}$, then $G$ is abelian which is not possible. If $|G / Z(G)|=21$, then $G / Z(G) \cong C_{7} \rtimes C_{3}$, and by Proposition 13 , $|\operatorname{Cent}(G)|=9$. If $|G / Z(G)|=9$, then $|\operatorname{Cent}(G)|=5$. If $|G / Z(G)|=27$, then by Proposition 12 , $|\operatorname{Cent}(G)|=11$ or 14 . Hence, $r \neq 6$ and so $|\operatorname{Cent}(G)|<r+4$. 
Now suppose $r=7$. By Remark 4, $G=X_{1} \cup X_{2} \cup \cdots \cup X_{7}$, and by Lemma 2, $\left|G: X_{2}\right| \leq 6$. Now if $\left|G: X_{2}\right|=5$, then $\mid G$ : $X_{1} \mid=3$ or 5 . If $\left|G: X_{1}\right|=3$, then by Remark $11, G=X_{1} X_{2}$, and by Proposition 6, $X_{1} \cap X_{2}=Z(G)$. Hence, $|G / Z(G)|=15$ which is not possible, since $G$ is nonabelian. If $\left|G: X_{1}\right|=5$, then $|G / Z(G)| \leq 25$, and since 5 is a divisor of $|G / Z(G)|$, then $|G / Z(G)| \in\{15,25\}$, which is again a contradiction. Hence, $r \neq 7$.

Now suppose $r=8$. By Remark 4, $G=X_{1} \cup X_{2} \cup \cdots \cup X_{8}$, and by Lemma 2, $\left|G: X_{2}\right| \leq 7$. Now if $\left|G: X_{2}\right|=3$, by Proposition 6, $|\operatorname{Cent}(G)|=5$, a contradiction. If $\left|G: X_{2}\right|=5$, then $\left|G: X_{1}\right|=3$ or 5 . If $\left|G: X_{1}\right|=3$, then by Remark 11, $G=X_{1} X_{2}$, and by Proposition 6, $X_{1} \cap X_{2}=Z(G)$. Hence, $|G / Z(G)|=15$ which is not possible, since $G$ is nonabelian. If $\left|G: X_{1}\right|=5$, then $|G / Z(G)| \leq 25$. Now since 5 is a divisor of $|G / Z(G)|,|G / Z(G)| \in\{15,25\}$, which is again a contradiction. Finally, suppose that $\left|G: X_{2}\right|=7$. By Lemma 2, $\left|G: X_{2}\right|=\left|G: X_{3}\right|=\cdots=\left|G: X_{8}\right|=7$. Therefore, $|G|=\sum_{i=2}^{8}\left|X_{i}\right|$, and by Theorem 10, $G=X_{1} X_{2}$. Again by Proposition 6, $X_{1} \cap X_{2}=Z(G)$. Since $|G|$ is odd, $\left|G: X_{1}\right|=3,5$, or 7. If $\left|G: X_{1}\right|=3$, then $|G / Z(G)|=21$. So $G / Z(G) \cong C_{7} \rtimes C_{3}$, and by Proposition 13 , $|\operatorname{Cent}(G)|=9$, which is a contradiction. If $\left|G: X_{1}\right|=5$, then $|G| Z(G) \mid=35$. Therefore, $G$ is abelian, which is not possible. If $\left|G: X_{1}\right|=7$, then $|G / Z(G)|=49$. Now by $[6$, Theorem 6$]$, $|\operatorname{Cent}(G)|=9$, which is a contradiction. Hence, $r \neq 8$.

Thus, $r=9$. By Lemma $7, G$ is a $C A$-group, and by Remark 4, $X_{i} \cap X_{j}=Z(G)$ for all distinct $i, j \in\{1,2, \ldots, 9\}$. Now $G=X_{1} \cup X_{2} \cup \cdots \cup X_{9}$, and by Lemma $2,\left|G: X_{2}\right| \leq 8$. If $\left|G: X_{2}\right|=3$, then $|\operatorname{Cent}(G)|=5$, a contradiction. If $\left|G: X_{2}\right|=5$, then $\left|G: X_{1}\right|=3$ or 5 . If $\left|G: X_{1}\right|=3$, then by Remark 11, $G=X_{1} X_{2}$. Since $X_{1} \cap X_{2}=Z(G),|G / Z(G)|=15$, which is a contradiction. If $\left|G: X_{1}\right|=5$, then $|G / Z(G)| \leq 25$, and since 5 is a divisor of $|G / Z(G)|,|G / Z(G)| \in\{15,25\}$, which is again a contradiction. If $\left|G: X_{2}\right|=7$, then $\mid G$ : $X_{1} \mid=3,5$, or 7. If $\left|G: X_{1}\right|=3$, then by Remark $11, G=X_{1} X_{2}$, and since $X_{1} \cap X_{2}=Z(G)$, therefore $|G / Z(G)|=21$ and so $G / Z(G) \cong C_{7} \rtimes C_{3}$, and by Proposition 13 , $|\operatorname{Cent}(G)|=9$, which is a contradiction. Similarly, If $\left|G: X_{1}\right|=5$, then $|G / Z(G)|=35$, a contradiction. Finally, if $\left|G: X_{1}\right|=7$, then $G=\left\langle X_{1}, X_{2}\right\rangle$. Therefore by Theorem $8,|G / Z(G)| \leq 64$. Since $|G|$ is odd and $G$ is nonabelian, $|G / Z(G)| \in\{21,35,49,63\}$. If $|G / Z(G)|=21$, then $G / Z(G) \cong C_{7} \rtimes C_{3}$, and by Proposition 13, $|\operatorname{Cent}(G)|=9$, which is a contradiction. If $|G / Z(G)|=35$, then $G$ is abelian which is not possible. If $|G / Z(G)|=49$, then by $[6$, Theorem 6$],|\operatorname{Cent}(G)|=9$, which is a contradiction. Now suppose that $|G / Z(G)|=63$. If $X_{1}$ or $X_{2}$ is normal in $G$, then $G=X_{1} X_{2}$. Since $X_{1} \cap X_{2}=$ $Z(G),|G / Z(G)|=49$, and by [6, Theorem 6], $|\operatorname{Cent}(G)|=$ 9 , a contradiction. It is easy to see that $C_{G}(g) / Z(G) \leq$ $C_{G / Z(G)}(g Z(G))$ for any $g \in G \backslash Z(G)$. Therefore, $\left|G: X_{i}\right|=$ 7,9 , or 21 , for $i \in\{1,2, \ldots, 9\}$. Let $x Z(G)$ be an element of order 7 in $G / Z(G)$. Then $C_{G / Z(G)}(x Z(G))$ is the normal Sylow 7-subgroup of $G / Z(G)$. Since $C_{G}(x) / Z(G) \leq C_{G / Z(G)}(x Z(G))$, $C_{G}(x) / Z(G)=C_{G / Z(G)}(x Z(G))$. Hence by [19, Lemma 3.1], $G$ has exactly one centralizer of index 9. Suppose $\left|G: X_{3}\right|=9$. Then $\left|G: X_{4}\right|=\left|G: X_{5}\right|=\left|G: X_{6}\right|=\left|G: X_{7}\right|=\left|G: X_{8}\right|=$ $\left|G: X_{9}\right|=21$ and $|G|>\sum_{i=2}^{9}\left|X_{i}\right|$, which is a contradiction by Theorem 10. Therefore, $\left|G: X_{3}\right|=7$. Similarly, we can show that $\left|G: X_{4}\right|=\left|G: X_{5}\right|=\left|G: X_{6}\right|=\left|G: X_{7}\right|=7$. So we have $\left|G: X_{1}\right|=\cdots=\left|G: X_{7}\right|=7,\left|G: X_{8}\right|=9$, and $\left|G: X_{9}\right|=21$. By $G=X_{1} \cup X_{2} \cup \cdots \cup X_{9}$ and the property $X_{i} \cap X_{j}=Z(G)$ for all distinct $i, j \in\{1,2, \ldots, 9\}$, it is easy to see that $|G|=\left|X_{1}\right|+\cdots+\left|X_{9}\right|-8|Z(G)|$. So we have $|G / Z(G)|<63$, a contradiction.

Now the proof of Theorem 1 is complete.

\section{Conflict of Interests}

The authors declare that there is no conflict of interests regarding the publication of this paper.

\section{References}

[1] A. Abdollahi, S. M. J. Amiri, and A. M. Hassanabadi, "Groups with specific number of centralizers," Houston Journal of Mathematics, vol. 33, no. 1, pp. 43-57, 2007.

[2] A. R. Ashrafi, "Counting the centralizers of some finite groups," The Korean Journal of Computational \& Applied Mathematics, vol. 7, no. 1, pp. 115-124, 2000.

[3] A. R. Ashrafi, "On finite groups with a given number of centralizers," Algebra Colloquium, vol. 7, no. 2, pp. 139-146, 2000.

[4] A. R. Ashrafi and B. Taeri, "On finite groups with a certain number of centralizers," Journal of Applied Mathematics \& Computing, vol. 17, no. 1-2, pp. 217-227, 2005.

[5] A. R. Ashrafi and B. Taeri, "On finite groups with exactly seven element centralizers," Journal of Applied Mathematics \& Computing, vol. 22, no. 1-2, pp. 403-410, 2006.

[6] S. M. Belcastro and G. J. Sherman, "Counting centralizers in finite groups," Mathematics Magazine, vol. 67, no. 5, pp. 366374, 1994.

[7] M. Zarrin, "Criteria for the solubility of finite groups by their centralizers," Archiv der Mathematik, vol. 96, no. 3, pp. 225-226, 2011.

[8] M. Zarrin, “On element-centralizers in finite groups," Archiv der Mathematik, vol. 93, no. 6, pp. 497-503, 2009.

[9] M. Zarrin, "On solubility of groups with finitely many centralizers," Bulletin of the Iranian Mathematical Society, vol. 39, no. 3, pp. 517-521, 2013.

[10] B. H. Neumann, "Groups covered by finitely many cosets," Publicationes Mathematicae Debrecen, vol. 3, pp. 227-242, 1954.

[11] M. J. Tomkinson, "Groups covered by finitely many cosets or subgroups," Communications in Algebra, vol. 15, no. 4, pp. 845859, 1987.

[12] G. Scorza, "Gruppi che possono pensarsi come somma di tre sottogrouppi," Bollettino della Unione Matematica Italiana, vol. 5, pp. 216-218, 1926.

[13] D. Greco, "Sui gruppi che sono somma di quattro o cinque sottogruppi," Rendiconto dell'Accademia delle Scienze Fisiche e Matematiche, vol. 23, pp. 49-59, 1956 (Italian).

[14] R. A. Bryce, V. Fedri, and L. Serena, "Covering groups with subgroups," Bulletin of the Australian Mathematical Society, vol. 55, no. 3, pp. 469-476, 1997.

[15] A. Abdollahi, M. J. Ataei, S. M. J. Amiri, and A. M. Hassanabadi, "Groups with a maximal irredundant 6-cover," Communications in Algebra, vol. 33, no. 9, pp. 3225-3238, 2005. 
[16] A. Abdollahi and S. M. J. Amiri, "On groups with an irredundant 7-cover," Journal of Pure and Applied Algebra, vol. 209, no. 2, pp. 291-300, 2007.

[17] N. Itô, "On finite groups with given conjugate types. I," Nagoya Mathematical Journal, vol. 6, pp. 17-28, 1953.

[18] J. H. E. Cohn, “On n-sum groups," Mathematica Scandinavica, vol. 75, no. 1, pp. 44-58, 1994.

[19] S. J. Baishya, "On finite groups with specific number of centralizers," International Electronic Journal of Algebra, vol. 13, pp. 5362, 2013. 


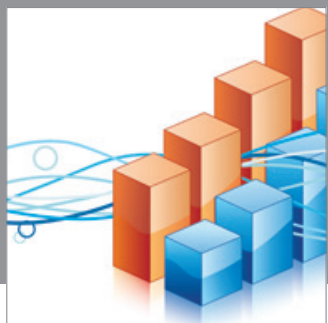

Advances in

Operations Research

mansans

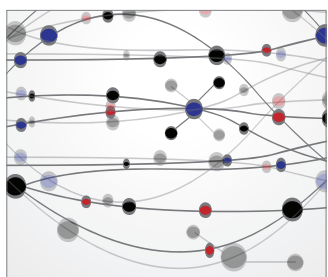

The Scientific World Journal
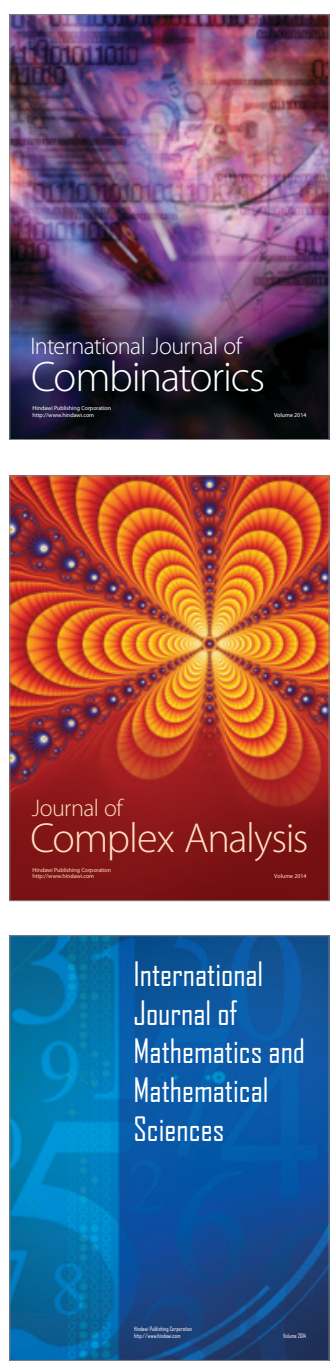
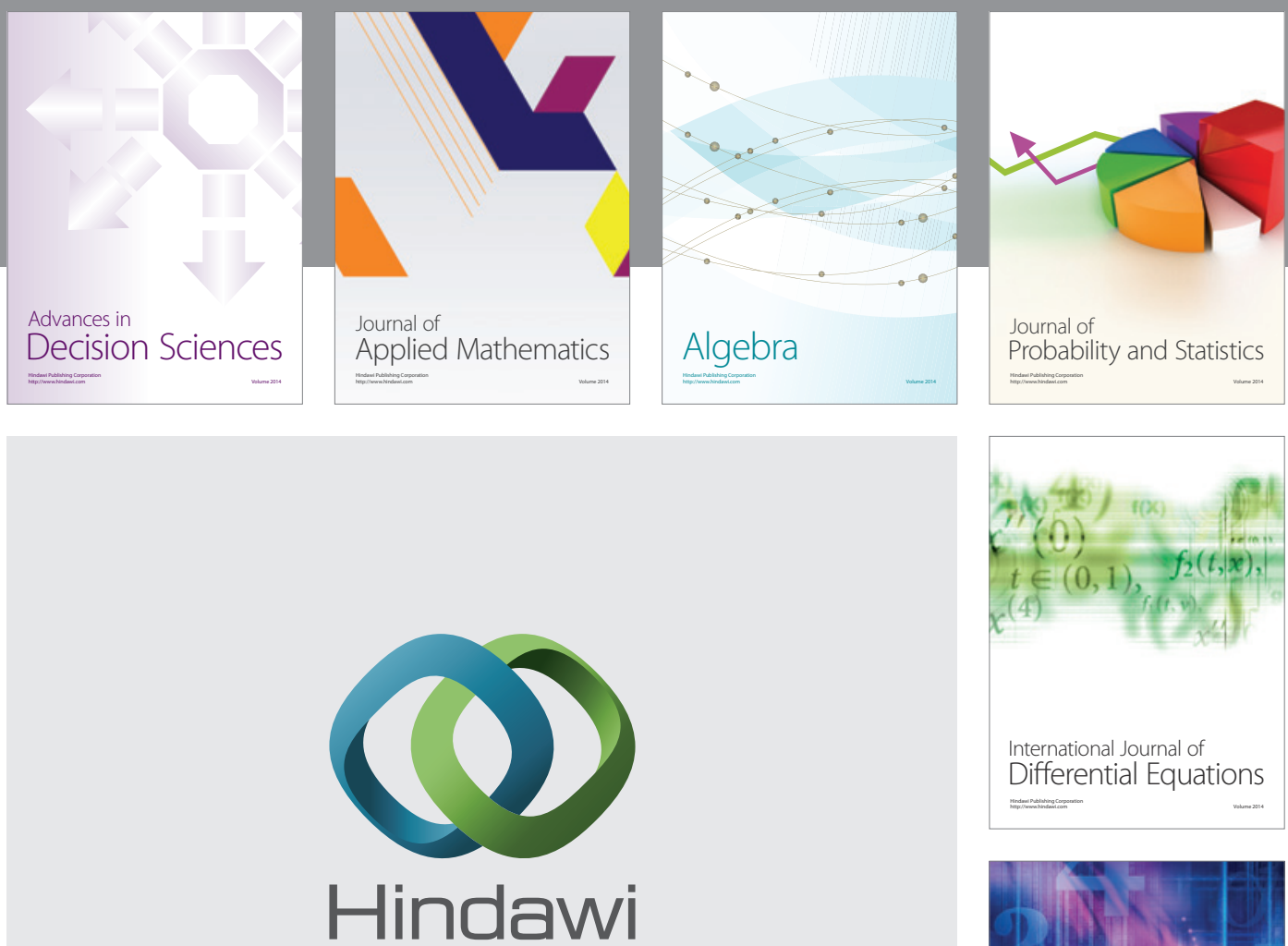

Submit your manuscripts at http://www.hindawi.com
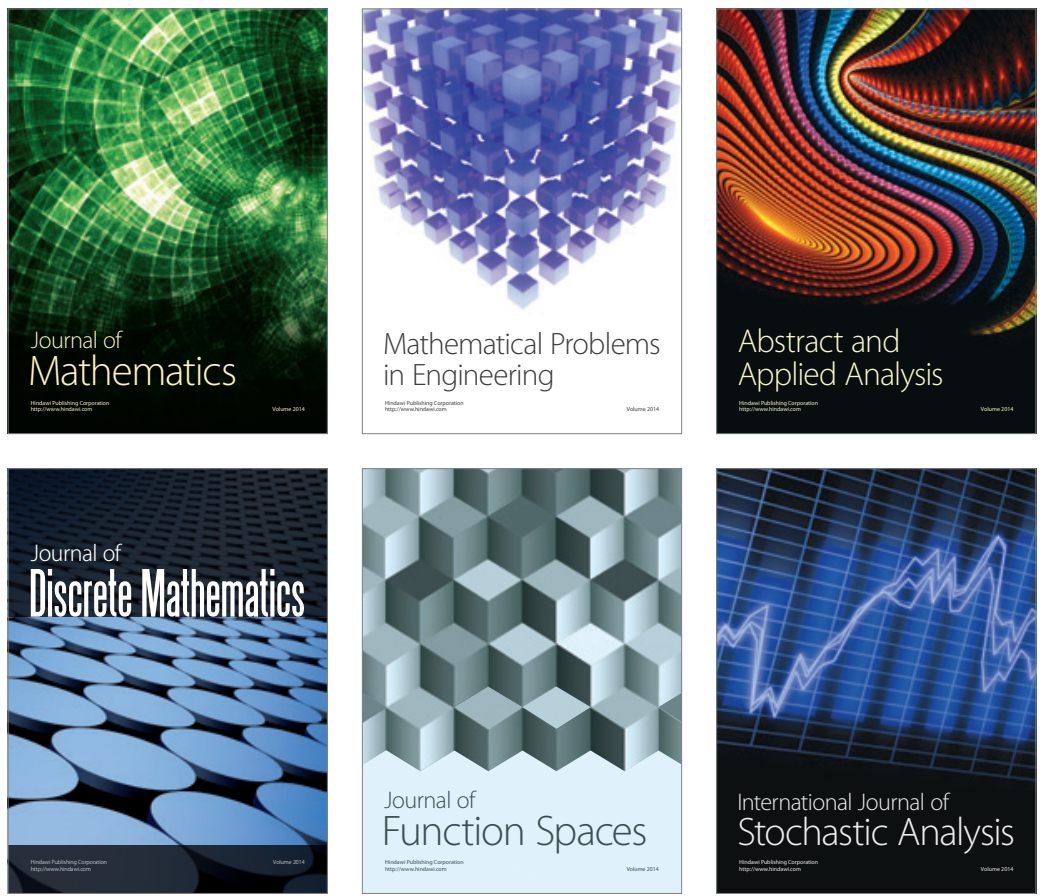

Journal of

Function Spaces

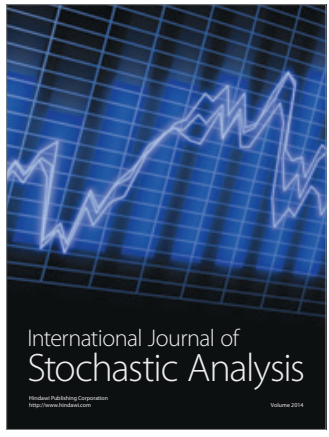

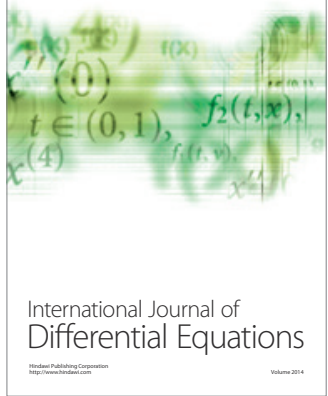
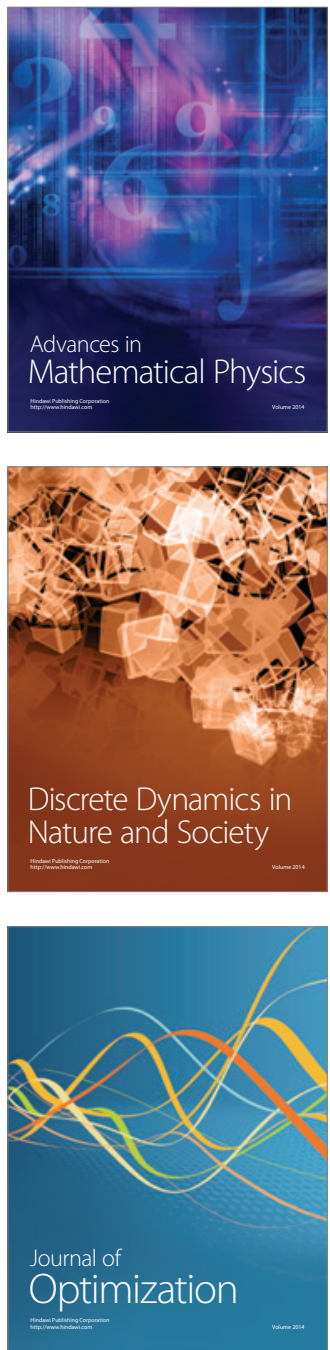